\title{
Trust Model Development for Cloud Environment using Fuzzy Mamdani and Simulators
}

\author{
Hamdy M. Mousa', Gamal F. Elhady ${ }^{2}$ \\ ${ }^{1}$ Faculty of Computers and Information's, Menofia University, Egypt \\ hamdimmm@hotmail.com \\ ${ }^{2}$ Faculty of Computers and Information's, Menofia University, Egypt \\ gamal.farouk@ci.menofia.edu
}

\begin{abstract}
Nowadays, Cloud computing is an expanding area in research and industry, which involves virtualization, distributed computing, internet, software, security, web services and etc. A cloud consists of several elements such as clients, data centers and distributed servers, internet and it includes fault tolerance, high availability, effectiveness, scalability, flexibility, reduced overhead for users, reduced cost of ownership, on demand services and etc. Now the next factor is coming, cost of Virtual machines on Data centers and response time. So this paper develop trust model in cloud computing based on fuzzy logic, to explores the coordination between Data Centers and user bound to optimize the application performance, cost of Virtual machines on Data centers and response time using Cloud Computing Analyst.
\end{abstract}

\section{Keywords}

Cloud Computing, Cloud Analyst, Trust Model, Fuzzy Logic Inference System (FIS).

\section{Council for Innovative Research}

Peer Review Research Publishing System

Journal: INTERNATION JOURNAL OF COMPUTERS AND TECHNOLOGY

Vol. 13, No. 11

editorijctonline@gmail.com

$\underline{\text { www.ijctonline.com, www.cirworld.com }}$ 


\section{INTRODUCTION}

Cloud services are becoming popular in terms of distributed technology because they allow cloud users to rent wellspecified resources of computing, network, and storage infrastructure. Cloud computing is a computing platform that resides in a large data center and is able to dynamically provide servers the ability to address a wide range of needs, ranging from scientific research. Cloud Computing can be defined as a model for enabling ubiquitous, convenient, ondemand network access to a shared pool of configurable computing resources (e.g., networks, servers, storage, applications, and services) that can be rapidly provisioned and released with minimal management effort or service provider interaction [17].

The services which offered by cloud computing are classified as: "X as a service (XaaS)" where X represents "Infrastructure", "Platform", "Software", "Database" etc. Among the various service models available in cloud, Infrastructure as a Service (laaS) plays a vital role. laaS is the delivery of computing resources as a service through APIs, which includes virtual machines, operating systems and other abstracted hardware [18]. The customer rents these resources which are dynamically scalable as per usage, rather than buying and installing them. Examples for laaS include Amazon and service providers. Due to the large scale and openness of these systems, a customer is often required to interact with service providers with whom he has few or no shared past interactions. To assess the risk of such interactions and to determine whether an unknown service provider is trustworthy, an efficient trust mechanism is necessary. Cloud is a highly agile and flexible utility computing paradigm that allows users to acquire virtualized computing resources in a payas-you go model [14]. Its merits, including no upfront investment, just-in-time resource provisioning, and fast deployment, is attracting more and more organizations to migrate their existing applications and develop new systems on cloud. Among all cloud service models, Infrastructure as a service (laaS) provides the most flexible service for users trying to deploy their own applications. To realize the benefits of using laaS cloud, users need to ensure the trading service providers can fully satisfy their applications' functional and non-functional requirements. However, there exists plenty of laaS cloud providers offering similar services with different pricings and performances, which creates difficulty for users to find the most suitable service. Therefore, it is essential to develop automatic systems to dynamically identify satisfactory. laaS services according to different application requirements [21]

Cloud computing is based on five attributes: multi-tenancy (shared resources), massive scalability, elasticity, pay as you go, and self-provisioning of resources, it makes new advances in processors, Virtualization technology, disk storage, broadband Internet connection, and fast, inexpensive servers have combined to make the cloud a more compelling solution. The main attributes of cloud computing are illustrated as follows:

- $\quad$ Multi-tenancy (shared resources): Cloud computing is based on a business model in which resources are shared (i.e., multiple users use the same resource) at the network level, host level, and application level.

- Massive scalability: Cloud computing provides the ability to scale to tens of thousands of systems, as well as the ability to massively scale bandwidth and storage space Elasticity: Users can rapidly increase and decrease their computing resources as needed.

- $\quad$ Pay as you used: Users to pay for only the resources they actually use and for only the time they require them.

- $\quad$ Self-provisioning of resources: Users self-provision resources, such as additional systems (processing capability, software, storage) and network resources. Cloud computing can be confused with distributed system, grid computing, utility computing, service oriented architecture, web application, web, broadband network, browser as a platform, Virtualization, and free/open software.

Cloud computing is a natural evolution of the widespread adoption of virtualization, service-oriented architecture, autonomic, and utility computing [3]. Details are abstracted from end-users, who no longer have a need for expertise in, or control over, the technology infrastructure "in the cloud" that supports them as shown in figure 1.

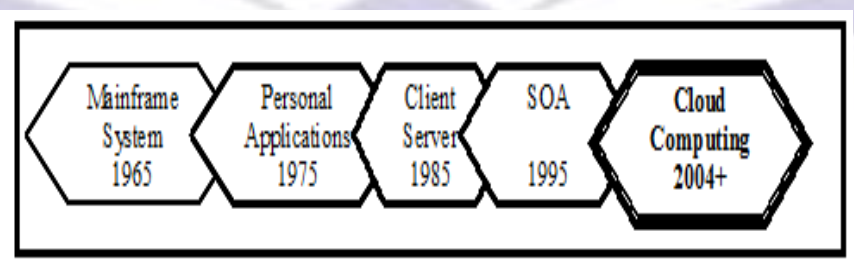

Fig1. Evolution of cloud computing

Cloud services exhibit five essential characteristics that demonstrate their relation to, and differences from, traditional computing approaches such as On-demand self-service, Broad network access, Resource pooling, Rapid elasticity, and Measured service [17[. Cloud computing often leverages Massive scale, Homogeneity, Virtualization, Resilient computing (no stop computing), Low cost/free software, Geographic distribution, Service orientation Software and Advanced security technologies.

The main objective of this paper is to develop trust model in cloud computing based on fuzzy logic. This paper explores the coordination between Data Centers and user bound to optimize the application performance, cost of Virtual machines on Data centers and response time by using a tool called Cloud Analyst. This can help users make an informed choice towards selecting the appropriate (Cloud Service Provider) CSP as per their requirement (Performance, Cost, Elasticity, Time and Security). 
The rest of the paper is organized as follows: Section 2 describes the previous related works and the cloud computing architecture is described in Section 3. Proposed Research Methodology and infrastructure details are described in Section 4. Section 5 explains the experimental results of Cloud Analyst. Paper conclusion and future work are present in Section 6 .

\section{PREVIOUS WORKS}

Cloud computing is defined as "a type of parallel and distributed system consisting of a collection of interconnected and virtualized computers that are dynamically provisioned and presented as one or more unified computing resources based on service-level agreements established through negotiation between the service provider and consumers" [14]. Different studies have been done in the area of cloud computing. Scientists and researchers in all over the world have a given different definition of cloud computing. The cloud model initially has focused on making the hardware layer consumable due to exponential growth in demand of computing and storage capacity. Different studies have been done in the area of cloud computing [1], have published a paper entitled "A barkeley view of cloud computing", compares general clouds with private data centers and concludes their pros and cons. Jaesun, Han [10], have published an article entitled "The Future of Cloud Computing and Server Platform", evaluates the performance of cloud and determines the impacts of cloud computing on organizational structure. Kim Won [11], have published an article entitled "Cloud Computing: Today and Tomorrow", discussed few essential options for data policy in cloud computing. Bryan Stephenson et al , [6] published a paper entitled "outsourcing business to cloud computing services". They studied about different kinds of users who are using various types of cloud computing services. Finally, they introduced the architecture for businesses, which desire to use cloud computing services by outsourcing business. Fang Hao et al [4], have published a paper entitled, "secure cloud" deals with the security issues and concerns in deploying cloud computing. Suhaidi Hassan, et al [13], have published a paper entitled "A survey on trust and trust management in cloud computing", analyzes the trust management systems recommended for cloud computing by various researchers with an unusual emphasis on their capability, their implementation and their applicability in the practical heterogenous cloud environment. Xiaodong Sun, et al [20], have published a paper entitled "A trust management model to enhance security of cloud computing environments", Discussed direct and recommended trust measurements based on fuzzy set theory. Their proposed model gives a helpful assess to improve security, robustness and fault tolerance of cloud computing. Thara Dillon, et al, [12], have published a paper entitled "A trust-evaluation metric for cloud applications", proposed a model for availability, usability, scalability and security parameters of trust for laaS using fuzzy-set theory.

In this paper, they developed an overall trust rating for a given CSPs based on mamdani fuzzy-inference system. We proposed a hierarchical model which extends the model described in [15], and rates the cloud service providers and their plans based on five parameters: Performance, Security, Elasticity, Time and Cost. In this model we developed the trust model in cloud computing based on Fuzzy Inference System. Fuzzy logic was used to provide trust in cloud computing Different types of attacks and trust models in service oriented systems, distributed system and so on are designed based on fuzzy logic system [5]. A formal trust management model for Software as a Service (SaaS) based on the basics of the trust characteristics is presented in [18]. This model is capable to handle various cloud services access scenarios where an entity may or may not have a past experience with the service.

\section{CLOUDCOMPUTING ARCHITECTURE}

\subsection{Cloud computing service models}

- Cloud Software as a Service (SaaS): Application and Information clouds, Use provider's applications over a network, cloud provider examples Zoho, Salesforce.com, Google Ap.

- Cloud Platform as a Service (PaaS): Development clouds, Deploy customer-created applications to a cloud, cloud provider examples Windows Azure, Google App Engine, Aptana Cloud [21].

- Cloud Infrastructure as a Service (laaS): Infrastructure clouds, Rent processing, storage, network capacity, and other fundamental computing resources, Dropbox, Amazon Web Services, Mozy, Akamai [21].

\subsection{Cloud computing deployment models}

- Private cloud : Enterprise owned or leased

- Community cloud: Shared infrastructure for specific community

- Public cloud: Sold to the public, mega-scale infrastructure

- Hybrid cloud: Composition of two or more clouds

\subsection{Cloud computing sub-services models}

- DataBase-as-a-Service (DBaaS): DBaaS allows the access and use of a database management system as a service.

- Storage-as-a-Service (STaaS): STaaS involves the delivery of data storage as a service, including database-like services, often billed on a utility computing basis, e.g., per gigabyte per month.

- Communications-as-a-Service (CaaS): CaaS is the delivery of an enterprise communications solution, such as Voice over IP, instant messaging, and video conferencing applications as a service [9]. 
- $\quad$ SECurity-as-a-Service (SECaaS): SECaaS is the security of business networks and mobile networks through the Internet for events, database, application, transaction, and system incidents.

- Monitoring-as-a-Service (MaaS): MaaS refers to the delivery of second-tier infrastructure components, such as log management and asset tracking, as a service, [9].

- Desktop-as-a-Service (DTaaS): DTaaS is the decoupling of a user's physical machine from the desktop and software he or she uses to work.

- Compute Capacity-as-a-Service (CCaaS): CCaaS is the provision of "raw" computing resource, typically used in the execution of mathematically complex models from either a single "supercomputer" resource or a large number of distributed computing resources where the task performs well.

\subsection{Cloud computing benefits}

Lower computer costs, improved performance, reduced software costs, instant software updates, improved document format compatibility, unlimited storage capacity, device independence, and increased data reliability

\subsection{Cloud computing drawbacks}

Requires a constant Internet connection, does not work well with low-speed connections, can be slow, features might be limited, stored data might not be secure, and stored data can be lost.

\subsection{Cloud Analyst}

The CloudAnalyst is built on top of CloudSim tool kit, by extending CloudSim functionality with the introduction of concepts that model Internet and Internet Application behaviors.

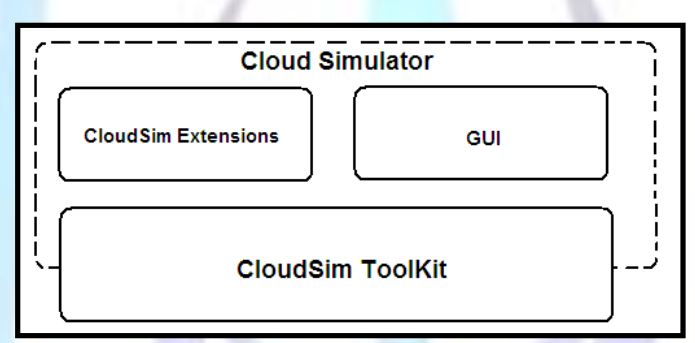

Fig 2. CloudAnalyst built on top of CloudSim toolkit

In the CloudAnalyst the world is divided in to six 'Regions' that coincide with the six main continents in the World. The other main entities such as User Bases and Data Centers belong to one of these regions. The simulator is developed $100 \%$ on Java platform, using Java SE 1.6, The GUI component is built using Swing components.

CloudSim features for modelling data centers is used in CloudAnalyst a User Base models a group of users that is considered as a single unit in the simulation and its main responsibility is to generate traffic for the simulation. An InternetCloudlet is a grouping of user requests. The number of requests bundled into a single Internet Cloudlet is configurable in Cloud Analyst. The Internet Cloudlet carries information such as the size of a request execution command, size of input and output files, the originator and target application id used for routing by the Internet and the number of requests. The Data Center Controller is probably the most important entity in the CloudAnalyst. A single Data Center Controller is mapped to a single cloudsim. Data Center object and manages the data center management activities such as VM creation and destruction and does the routing of user requests received from User Bases via the Internet to the VMs [19].

\section{RESEARCH METHODOLOGY}

Our proposed approach includes: Problem Definition, Data Collection, Trust Model, Parameters Model, Data Collection and Fuzzy Design Model. Figure 3, show that the research approach. The next following sections describe the research steps.

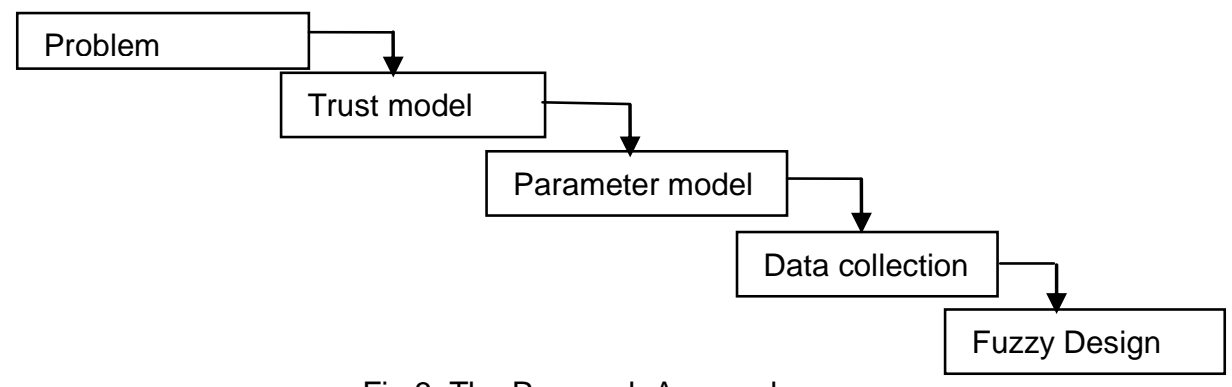

Fig 3. The Research Approach 


\subsection{Problem Definition}

On the cloud computing system, there is no framework which a cloud service consumer can make an intelligent trustbased decision regarding service selection from a service provider. Given the potential growth of cloud computing and the business implications, it is very important to have such architecture in place. The primary issues which are not investigated in the related literature are:

- The difficulties faced by cloud users when they want to sign online agreements with cloud providers; there is no clear and reliable method for selecting the most suitable parameters for the contracts.

- The lack of a trust model to calculate and estimate the cost for each level of the cloud architecture.

- $\quad$ Although trust and reputation systems have been widely proposed and implemented for various types of online services, no such models have been proposed for cloud computing; cloud users also need such systems in order to select the most trustworthy of services that are already being offered by cloud providers.

In this paper, we will concentrate on how to develop and evaluate the trusted cloud providers model in such a way that users of cloud can easily understand and start.

\subsection{Trust Management Model}

Trust is strongly connected to confidence and it implies some degrees of uncertainly, hopefulness or optimism [7]. The Trust Model concentrates on the measure of trust value based on direct experience which is considered as direct trust and Recommended information which is, for CSP in Inter Domain and Intra Domain. Figure 4, shows the trust model architecture of the trust assessment.

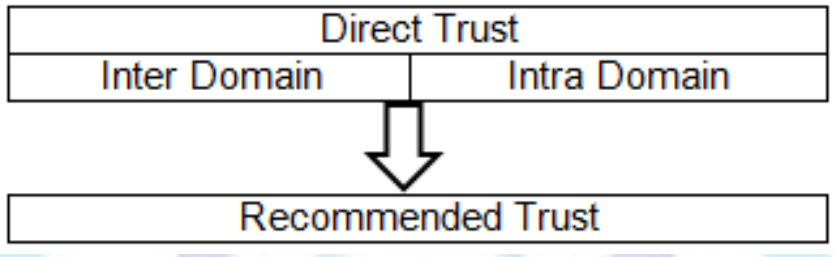

Figure 4. Trust Model

In our paper, we have simulated an environment using Cloud Analyst and focused on the estimation of Inter Domain trust value for CSP based on the direct relationship. Cloud Analyst is developed by, [19]. It is built on top of CloudSim and separates the simulation experimentation from a programming task enabling one to concentrate on the simulation parameters rather than the technicalities of programming. Simulation in Cloud Analyst involves the following steps:

1. Defining and configuration of User Bases.

2. Defining and configuring Data Centers

3. Allocating of Virtual Machines in Data Centers.

4. Review and Adjustment of various other parameters such as Packet size, No of packets, Bandwidth, and Load balancing policies

The Cloud Analyst enables us to model different scenarios of CSPs and User Bases, and provides a comprehensive output detailing the response time, Data Center processing time and total cost involved in the communication and computation.

\subsection{Parameter Model}

Some attributes are used to be performing parameter model this attributes are defined by Service Measurement Index (SMI) . As the following: Security, Elasticity, Performance, Cost and Time. Each of these attributes consisting a set of Key Performance Indicators (KPIs), which explain the data to be collected for measurement. KPIs are experimental measurements, agreed to earlier, that reveals the vital success factors of an organization. They will fluctuate depending on the organization. Based on the KPI that frame the attributes in evaluating the trust and user satisfaction. We discussed performance, cost, elasticity, security and time in this paper. Table 1 shows the Factors Impact of Trust Degree (FITD) and there KPIs Attribute [11]. 
Table 1. Parameters Model

\begin{tabular}{|c|l|}
\hline FITD & \multicolumn{1}{|c|}{ KPIs Attribute } \\
\hline Performance & $\begin{array}{l}\text { Accuracy, Functionality, Stability, } \\
\text { Interoperability, Service Response Time }\end{array}$ \\
\hline Elasticity & $\begin{array}{l}\text { Adaptability, Capacity, Extensibility, Flexibility, } \\
\text { Portability, Scalability }\end{array}$ \\
\hline Cost & $\begin{array}{l}\text { Acquisition and training cost, Ongoing cost, } \\
\text { Profit or Cost Sharing }\end{array}$ \\
\hline Time & $\begin{array}{l}\text { User Base Response Time. } \\
\text { User Base Request Serving Time. }\end{array}$ \\
\hline Security & Data location, Data Recovery \\
\hline
\end{tabular}

In order to evaluate the trust value for CSP comprises of two stages as shown in Figure 5 . The first stage is the implementation with the help of Mamdani Fuzzy Inference System [8]. It takes Performance, Cost, Elasticity and Time as inputs and produces a range of values which could be easily fed as input to the next level of processing. The Performance attribute can be evaluated by Processor Speed and Number of Processors as inputs to the Mamdani FIS. Cost attribute is calculated with the following inputs: Virtual Machine (V.M) Cost, Memory Cost, Storage Cost, Data Transfer Cost. Elasticity attribute has number of Physical Units, Memory Size, number of V.Ms as its inputs. Time attributes are User Base (UB) Response Time, User Base (UB) request and servicing times as inputs. Finally, Security attribute include Data Location and Recovery as inputs to the Mamdani Fuzzy based Inference System.

The second stage is the implementation using also Mamdani FIS. It takes the output of the first stage and helps to obtain the trust rating for the CSP.

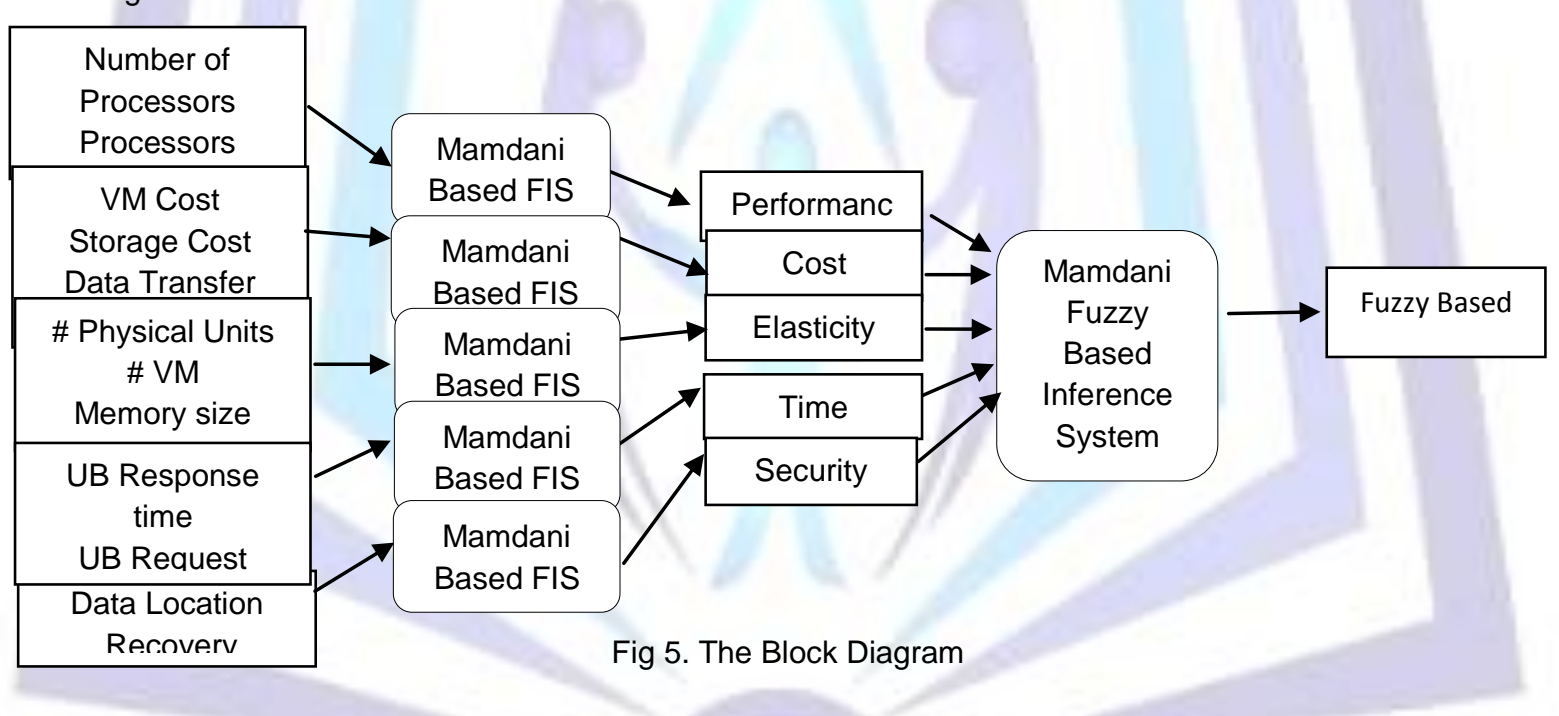

For the Mamdani FIS, the membership values for performance, Cost, Elasticity, Time and Security, parameters are assumed as low, medium, high and very high as per the requirement. For example, certain input parameters can have values only in a short interval while some may vary over larger range. The above two stages are implemented hierarchically using the fuzzy logic blocks in Simulink of Matlab [8].

\subsection{Data collection}

Data collection is considered one of the most important step in the development of fuzzy-based control system. So, the model with a fuzzy inference approach must be trained with training data that represent the greatest possibilities of application [2]. In our study, we use the data which was collected from cloud computing experts and cloud users. By an online-based survey was developed in order to collect more data sets from different locations. The survey with the designed research questions was conducted to collect values for the most important variables which had already been selected to present the trust value in a cloud-based application.

\subsection{Fuzzy Logic Model Design}

To estimate the trust value of the cloud service providers using fuzzy logic model is described in [15]. This model is used as to extend the mathematics ontology in certain method with fuzziness in order to help make an intelligent decision. The proposed fuzzy logic method in this paper uses three fuzzy sets for the input factors and four fuzzy sets for the parameters of output. The three fuzzy sets which are low $(\mathrm{L})$, medium $(\mathrm{M})$ and high $(\mathrm{H})$ are used to characterize the fuzzy value for 
each input which are Performance, cost, security, Elasticity and Time. The fuzzy sets that represent the output parameters are: poor $(P)$, good $(G)$, very good $(V G)$ and excellent $(E)$. In this model, Security was considered as important parameter which is requirement for the users of the cloud since when a company outsources its confidential data to another company or a cloud; it needs assurance that the service provider has used "reasonable security" to protect those data [16]

\section{EXPERIMENT Results}

In this section we show our methodology for verifying the proposed trust calculation model for cloud-based online services. The implementation of the proposed model is provided with the final results of the experiment.

\subsection{Cloud setup Simulation and Analyst}

To perform the simulation some of the User Bases, Cloud Service Providers and data center at different reigon are to be set. We define six user bases representing the six main regions of the world with parameters are described in Table 2. The simulation depend on a given parametes such as: cost, Time and virtual machine.

Table 2. User base region

\begin{tabular}{|l|l|l|}
\hline $\begin{array}{c}\text { User } \\
\text { base }\end{array}$ & $\begin{array}{c}\text { Cloud } \\
\text { Service } \\
\text { Providers }\end{array}$ & \multicolumn{1}{|c|}{ Region } \\
\hline UB1 & CSP-A & $0-$ N. America \\
\hline UB2 & CSP-B & $1-$ S. America \\
\hline UB3 & CSP-C & 2 - Europe \\
\hline UB4 & CSP-D & 3 - Asia \\
\hline UB5 & CSP-E & 4 - Africa \\
\hline UB6 & CSP-F & $5-$ Ocenia \\
\hline
\end{tabular}

Many of scenarios are used to setup cloud simulation. The user bases remain constant across the scenarios whereas the CSPs setup can be changes. So for the same amount of user load we are able to determine the performance of various CSPs. We take an example scenario with six different CSPs each with unique setting representing the geographic diversity, the cost factor, and the processing capabilities. Table 3, show the values which are taken to simulate the cloud environment by Cloud Analyst. So, six different simulations are running and each produces an output report detailing the Total Cost, the Response time, Data Center Request Servicing Times and the User Base Request Servicing Times.

Table 3. Simulation setup

\begin{tabular}{|c|c|c|c|c|c|c|c|c|c|c|}
\hline \multirow{2}{*}{$\begin{array}{l}\text { Cloud } \\
\text { Service } \\
\text { Provider }\end{array}$} & \multicolumn{2}{|c|}{ Virtual Machine } & \multicolumn{4}{|c|}{ Cost } & \multicolumn{4}{|c|}{ Data Center } \\
\hline & $\begin{array}{c}\# \\
\text { VMs }\end{array}$ & $\begin{array}{c}\text { Memory } \\
\text { (MB) }\end{array}$ & $\begin{array}{c}\text { Cost Per } \\
\text { BW (Data } \\
\text { transfer) } \\
(\$ / G B)\end{array}$ & $\begin{array}{c}\text { Cost Per } \\
\text { Storage } \\
(\$ / \mathbf{s})\end{array}$ & $\begin{array}{l}\text { Cost Per } \\
\text { Memory } \\
(\$ / s)\end{array}$ & $\begin{array}{c}\text { Cost Per } \\
\text { VM } \\
(\$ / H r)\end{array}$ & $\begin{array}{c}\text { \#Physical } \\
\text { units }\end{array}$ & $\begin{array}{c}\text { Processor } \\
\text { Speed }\end{array}$ & $\begin{array}{c}\# \\
\text { processors } \\
\text { per } \\
\text { machine }\end{array}$ & Region \\
\hline CSP-A & 200 & 512 & 0.15 & 0.15 & 0.05 & 0.4 & 6 & 100000 & 24 & 0-N.America \\
\hline CSP-B & 150 & 1024 & 0. & 0.25 & 0.05 & 0.1 & 3 & 120000 & 400 & 1-S.America \\
\hline CSP-C & 50 & 1024 & 0.02 & 0.08 & 0.05 & 0.05 & 6 & 80000 & 68 & 2-Europe \\
\hline CSP-D & 5 & 512 & 0.05 & 0.25 & 0.05 & 0.8 & 7 & 10000 & 12 & 3-Asia \\
\hline CSP-E & 30 & 1024 & 0.2 & 0.15 & 0.05 & 0.5 & 3 & 60000 & 4 & 4-Africa \\
\hline CSP-F & 125 & 512 & 0.09 & 0.1 & 0.05 & 0.3 & 3 & 160000 & 16 & 5-Ocenia \\
\hline
\end{tabular}


Figures 6, 7 are showing the simulation in progress during running and after simulation complete running. In this figures, UB represents User Bases and DC represents Data Centers.

Simulation Running...

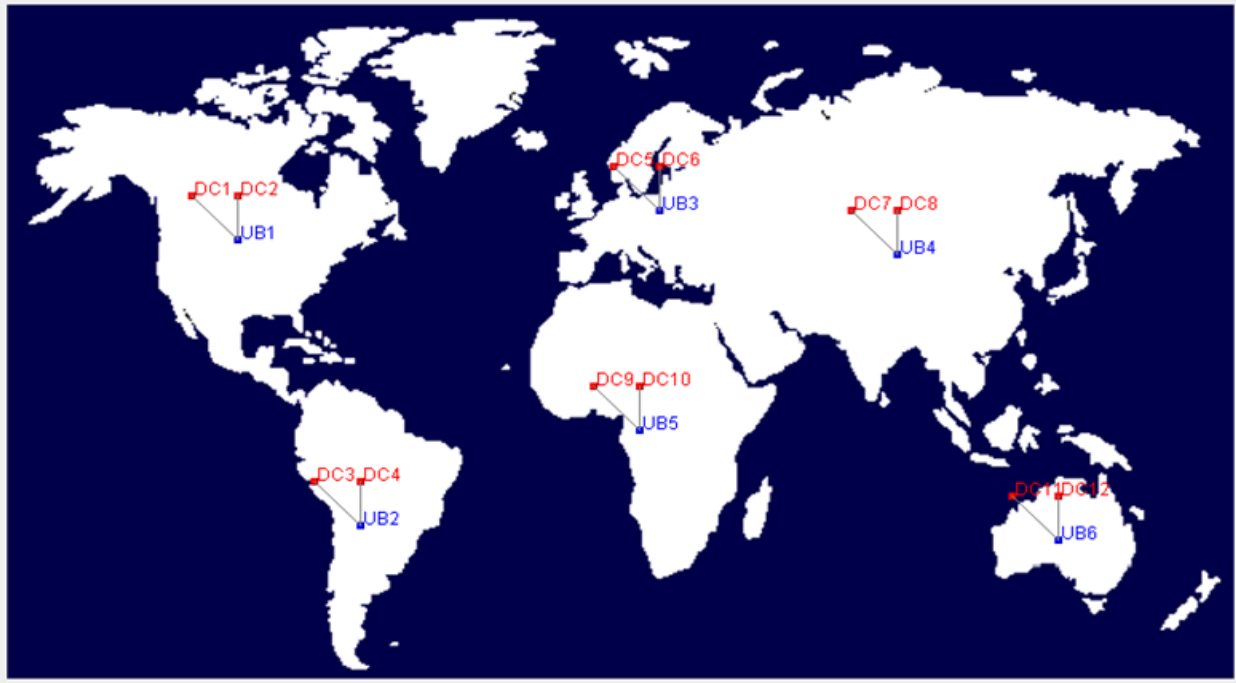

Fig 6. The Cloud Analyst Simulator during Running

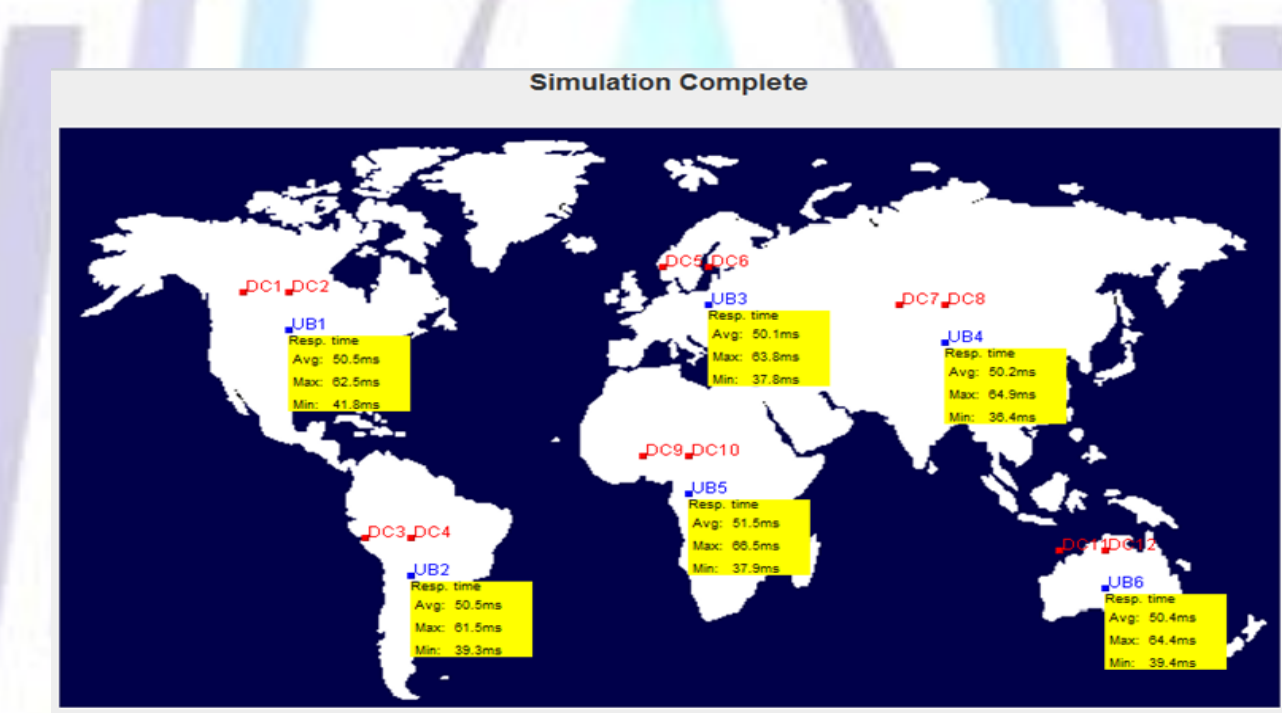

Fig 7. The Cloud Analyst Simulator after Running

The results of the Cloud Analyst for the assumed scenario can be summarizes in Table 3 . The Response times and Data Center Request Processing times, User Base Request Servicing Times with the Total Cost factor in \$. Table 4, show that CSP-C is cheaper compared to other CSPs. Next section 5.2 describes the estimation of trust value from these results for each CSP using Fuzzy Logic.

Table 4. Cloud Analyst Simulations Results

\begin{tabular}{|c|c|c|c|c|c|c|c|}
\hline \multirow{2}{*}{$\begin{array}{l}\text { Cloud } \\
\text { Service } \\
\text { Provider }\end{array}$} & \multicolumn{2}{|c|}{$\begin{array}{l}\text { Response } \\
\text { Time } \\
\text { (ms) }\end{array}$} & \multicolumn{2}{|c|}{$\begin{array}{l}\text { DC } \\
\text { Request } \\
\text { Servicing } \\
\text { Times } \\
\text { (ms) }\end{array}$} & \multicolumn{2}{|c|}{$\begin{array}{l}\text { UB } \\
\text { Request } \\
\text { Servicing } \\
\text { Times } \\
\text { (ms) }\end{array}$} & \multirow{2}{*}{$\begin{array}{c}\text { Total } \\
\text { Cost } \\
\$\end{array}$} \\
\hline & $\begin{array}{l}\text { Min } \\
\text { (ms) }\end{array}$ & $\begin{array}{l}\text { Max } \\
\text { (ms) }\end{array}$ & $\begin{array}{l}\text { Min } \\
\text { (ms) }\end{array}$ & $\begin{array}{l}\text { Max } \\
\text { (ms) }\end{array}$ & $\begin{array}{l}\text { Min } \\
\text { (ms) }\end{array}$ & $\begin{array}{l}\text { Max } \\
\text { (ms) }\end{array}$ & \\
\hline CSP-A & 42.03 & 63.83 & 0.01 & 2.08 & 0.02 & 3.11 & 160.52 \\
\hline CSP-B & 39.36 & 61.52 & 0.01 & 1.03 & 0.02 & 1.63 & 30.75 \\
\hline
\end{tabular}




\begin{tabular}{|l|r|r|r|r|r|r|l|}
\hline CSP-C & 37.77 & 63.76 & 0.02 & 0.86 & 0 & 1.54 & 5.52 \\
\hline CSP-D & 36.36 & 64.61 & 0 & 0.77 & 0.02 & 1.72 & 8.82 \\
\hline CSP-E & 37.75 & 64.4 & 0 & 0.77 & 0.19 & 2.17 & 31.61 \\
\hline CSP-F & 39.03 & 64.02 & 0 & 0.77 & 0.01 & 1.55 & 47.11 \\
\hline
\end{tabular}

The fuzzy logic toolbox of Matlab is used to design and implement our model. This toolbox includes ready functions and calculation methods to implement one type of fuzzy inference systems such as the Mamdani inference system. In our model, we use the Mamdani fuzzy methods with gbell membership function for inputs and output.

\subsection{Fuzzy Logic Implementation}

Performance: Performance use two input values number of processors and the processor speed as shown in Figure 5 . Both have three member functions each, low, Medium, High. See figures, 8, and 9.

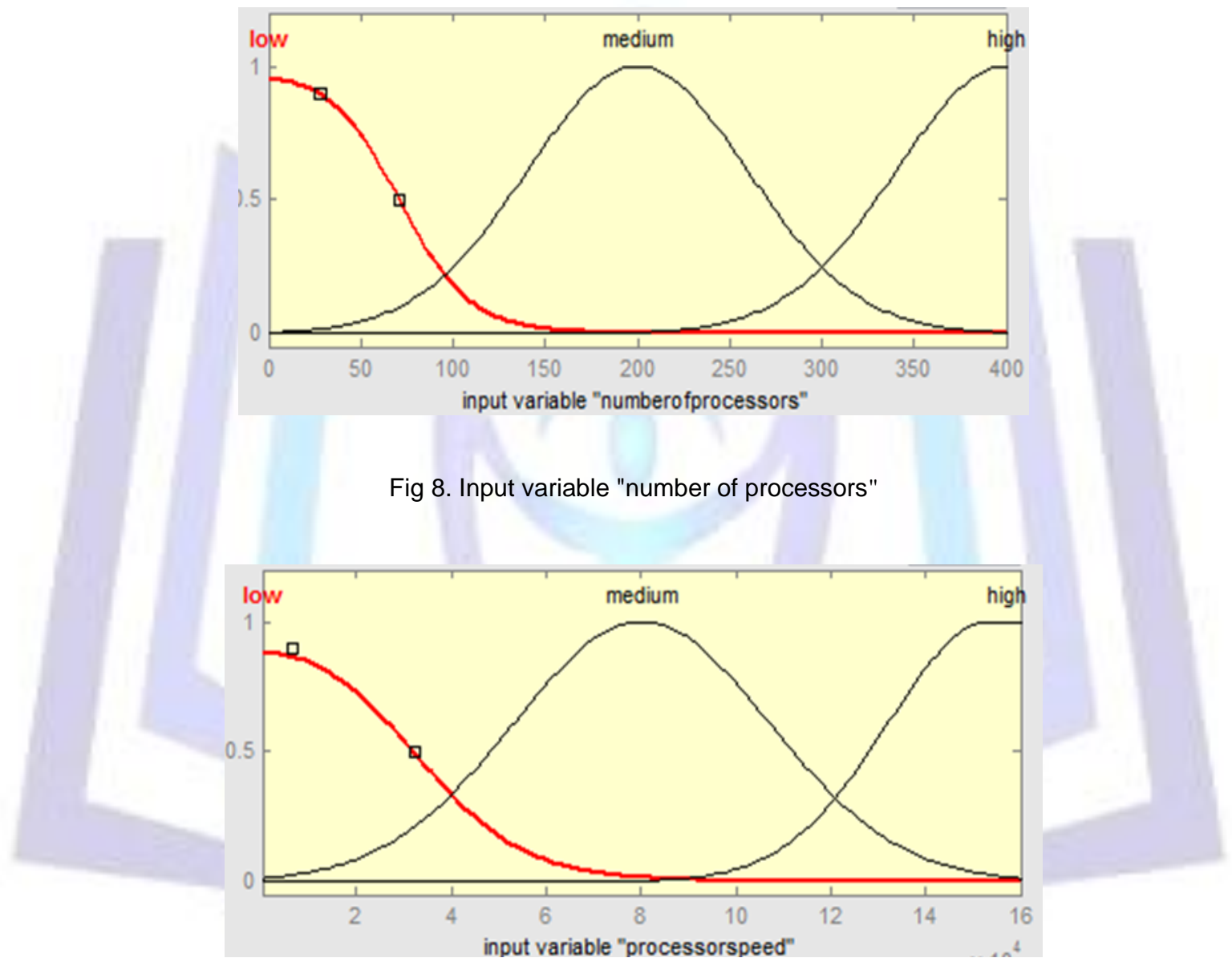

Fig 9. Input variable "processor speed"

The output of "performance" has five member functions: very low, low, medium, high, and very high. The outputs obtained from the FIS are CSP A-Low, CSP B-Medium, CSP C-Medium, CSP D- Medium, CSP E- Very High and CSP F-Low. The total of nine rules is written. Figure 10, shows the sample Rule Viewer when implemented in Matlab 


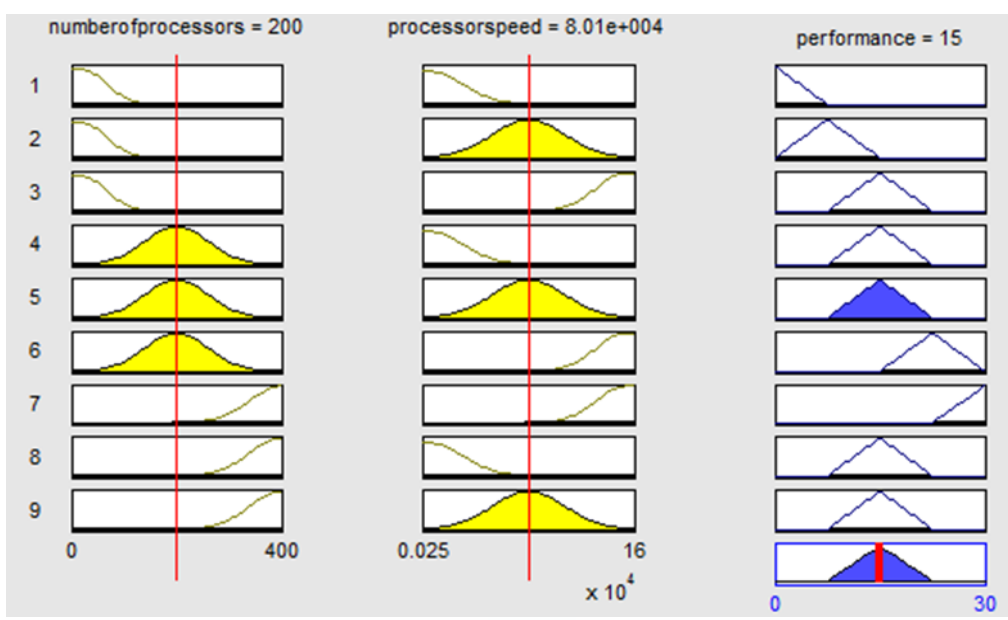

Fig 10. Performance Rule Viewer

Cost: The block Cost also comprises of four inputs V.M cost, Storage cost, Data Transfer cost and Processing Cost. The output of "Cost" has three member functions low, medium and high. Here a total of thirty four rules are written see figure 11.

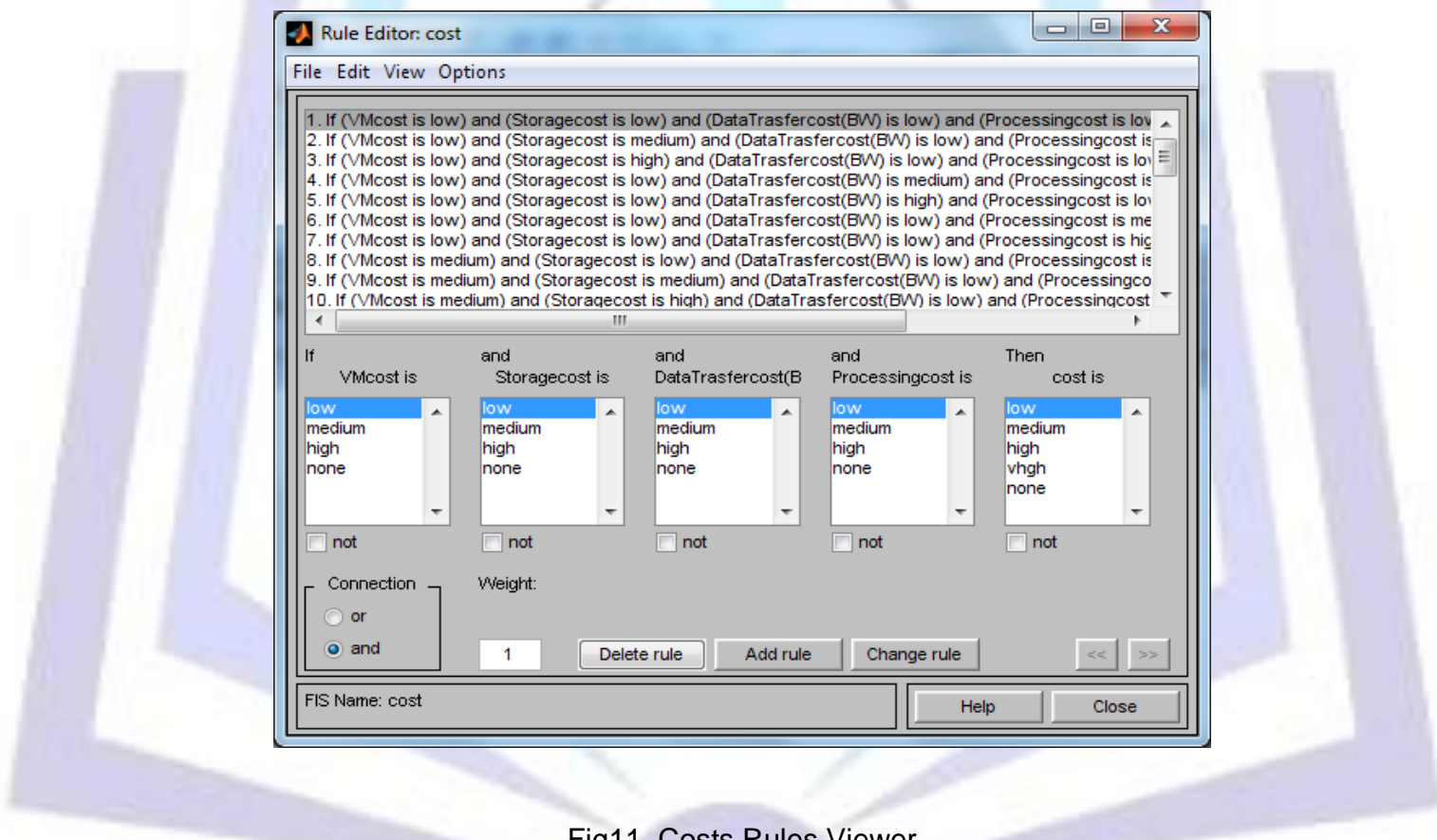

Fig11. Costs Rules Viewer

At given values of CSPs to the Cost model we get results as CSP A-High, CSP B-Medium, CSP C- High, CSP D-High, CSP E- High and CSP F-Medium.

Elasticity: the Elasticity model has three input parameters mapped to one output. Each of the input parameters in Figure 5 has different membership functions. "Physical units" has two member functions while "Memory" and "V.M" have three member functions each. The range of member functions is chosen based on the actual range of values used. A total of eight rules are written. The output of "Elasticity" has 3 member functions low, medium and high. When the input related to CSP A is fed to the Matlab FIS, the Elasticity comes out as Medium. Similarly for CSP B it is medium, CSP C-low, CSP Dmedium, CSP E-medium, CSP F-medium.

Security: Security also has two input values namely data location and recovery. Both of the input parameters in figure 5 have three membership functions. The output "security" has three membership functions low, medium and high. The outputs obtained from the FIS are CSP A- Low, CSP B- Medium, CSP C- Low, CSP D- Low, CSP E- High, CSP F- High.

Time: Time has two input values namely User Base Response Time, and User Base Request Serving Time. Both have three membership functions each. The output "Time" has three membership function low, medium, and high. The outputs obtained from FIS are CSP A- Low, CSP B- Medium, CSP C- Low, CSP D- Medium, CSP E- Medium, CSP F- High. 
The main models for the fuzzy logic system are shown in figure 12. We used FIS editor in Matlab to develop the model. The proposed model was implemented with five input factors: Performance, Cost, Elasticity, Security and Time. These five inputs are directed as inputs to the fuzzy inference system implemented with the Mamdani method. The Mamdani fuzzy model using FIS, and rules to execution and provide a trust rating. Assuming equal weights for all the rules the trust values estimated for each $\mathrm{CSP}_{\mathrm{s}}$ from above described conceptual model are classified in Table 5.

Table 5. Trust Values of $\mathrm{CSP}_{\mathrm{s}}$

\begin{tabular}{|l|l|l|l|l|l|l|l|}
\hline \multirow{2}{*}{$\begin{array}{l}\text { Cloud } \\
\text { Service } \\
\text { Provider }\end{array}$} & \multicolumn{3}{|l|}{ Five Parameters in Direct and Recommended Trust } & \multicolumn{2}{l|}{ Trust result } \\
\cline { 2 - 8 } & $\begin{array}{l}\text { Perfor-- } \\
\text {-mance }\end{array}$ & Cost & Elasticity & Security & Time & $\begin{array}{l}\text { Trust } \\
\text { values }\end{array}$ & $\begin{array}{l}\text { Trust } \\
\text { rating }\end{array}$ \\
\hline CSP-A & 0.499 & 0.222 & 0.321 & 0.050 & 0.350 & 0.456 & Good \\
\hline CSP-B & 0.999 & 0.441 & 0.642 & 0.525 & 0.500 & 0.910 & Excellent \\
\hline CSP-C & 0.749 & 0.343 & 0.481 & 0.787 & 0.500 & 0.682 & V Good \\
\hline CSP-D & 0.350 & 0.156 & 0.225 & 0.367 & 0.400 & 0.364 & Poor \\
\hline CSP-E & 0.399 & 0.445 & 0.258 & 0.525 & 0.306 & 0.406 & Good \\
\hline CSP-F & 0.649 & 0.511 & 0.417 & 0.682 & 0.650 & 0.638 & V Good \\
\hline
\end{tabular}

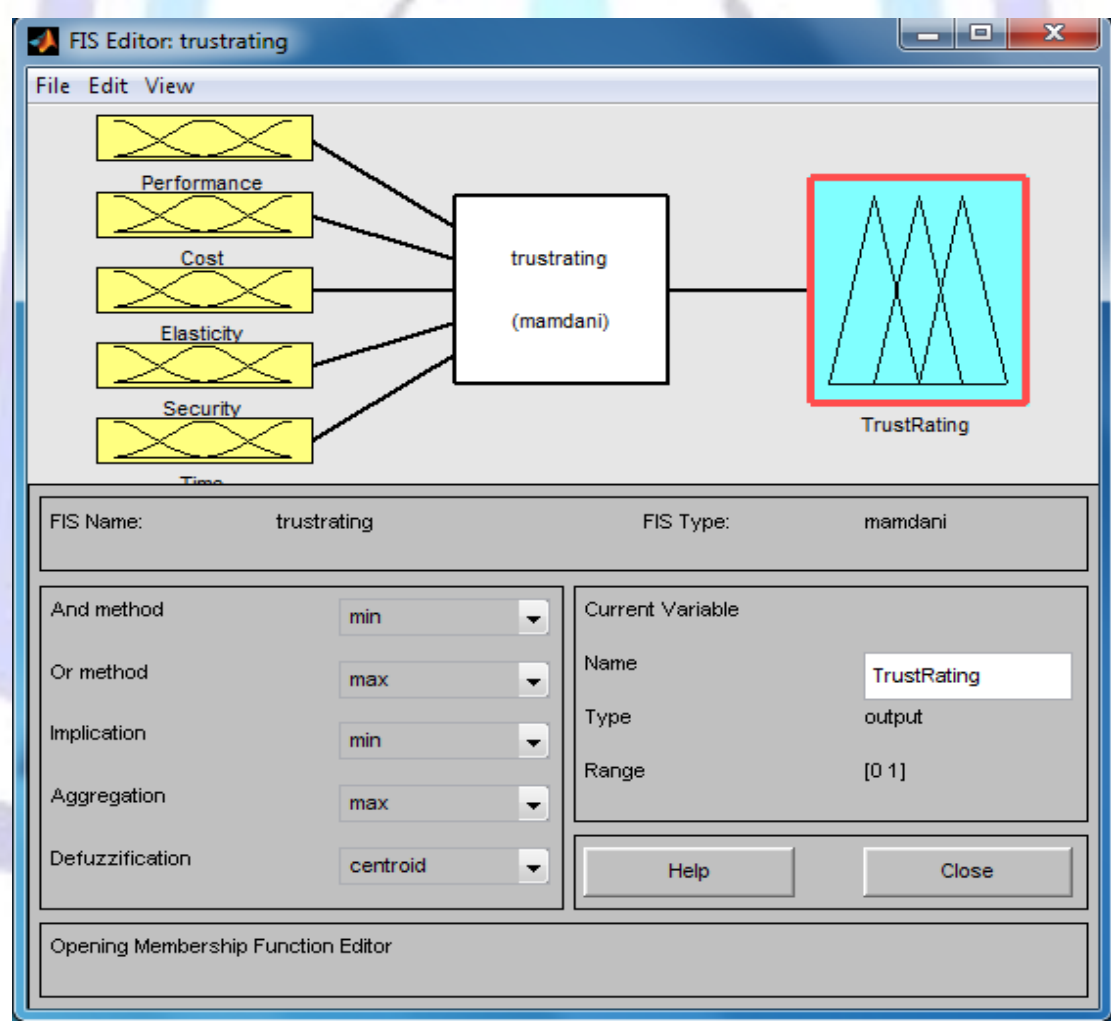

Fig 12. FIS Editor Interface.

To verify the efficient of our proposed model, the experimental results are done for comparison between our model and other model described in [15]. The proposed model more than efficient and accuracy, where, we uses five parameters such that, Performance, Security, Elasticity, Time and Cost than other model uses three parameters, Agility, Financial and Performance for developed the trust model in cloud computing based on Fuzzy Inference System. 


\section{CONCLUSION AND FUTURE WORK}

This paper used to developed trust model to evaluation the trust values based on fuzzy logic system. This method enables cloud users to evaluate the trustworthiness of cloud services providers. I took an example scenario with six different CSPs each with unique setting representing the geographic diversity, the cost factor and the processing capabilities. So, six different simulations are run using cloud analyst and each produces an output report detailing, the response time, data center processing time, user base request servicing time and the total cost with the factor in $\$$. Based on this report the CSP C is cheaper Compared to other CSPs. Also, I have revealed that Inter Domain direct trust value for CSPs can be estimated using fuzzy logic tool box, which can serve as an indicator for the users to choose a CSP as per their requirement. Our future work can be extends to others parameters and User Bases at different region.

\section{REFERENCES}

[1] Armbrust, M., et al. Above the clouds: A Berkeley view of cloud computing. Tech.Rep. UCB/EECS-2009-28, EECS Department, U.C. Berkeley, Feb 2009.

[2] C. Yin, et al., "Methods to improve prediction performance of ANN models," Simulation Modelling Practice and Theory, vol. 11, pp. 211-222, 2003.

[3] C. Qu and R. Buyya"A Cloud Trust Evaluation System using Hierarchical Fuzzy Inference System for Service Selection"IEEE 28th International Conference on Advanced Information Networking and Applications, 2014.

[4] Fang Hao, T. V. Lakshman, Sarit Mukherjee, Haoyu Song, Bell Labs, Alcatell Lucent, "Secure cloud computing with a virtualized network infrastructure" HotCloud'10 Proceedings of the 2nd USENIX conference on Hot topics in cloud computing, page 16-16.

[5] Florian Skopik, Daniel Schall, Schahram Dustdar, "Trustworthy Interaction Balancing in Mixed Service-oriented Systems", ACM 2010, SAC'10 March 22-26, 2010, Sierre, Switzerland

[6] Hamid R. Motahari-Nezhad, Bryan Stephenson, Sharad Singha, "Outsourcing Business to Cloud Computing Services: Opportunities and Challenges", IEEE IT Professional, Special Issue on Cloud Computing, Vol. 11, No. 2. (September 2009) Key: citeulike:4453620.

[7] H. M. Alabool and A. K. Mahmood, "Trust-based service selection in public cloud computing using fuzzy modified vikor method," Australian Journal of Basic and Applied Sciences, vol. 7, no. 9, pp. 211-220, 2013.

[8] http://www.mathworks.com/help/pdf_doc/fuzzy/fuzzy.pdf" Fuzzy Logic Toolbox ${ }^{T M}$ User"s Guide.

[9] J. Muller, et al., "Customizing Enterprise Software as a Service Applications: Back-End Extension in a Multi-tenancy Environment," 2009, p. 66.

[10] Jaesun Han: "The Future of Cloud Computing and Server Platform", http://www.web2hub.com/blog/82 (in Korean) February 1, 2008.

[11] Kim Won, Cloud Computing : "Today and Tomorrow", 2009.

[12] Mohammed Alhamad, Tharam Dillon and Elizabeth Chang. "A Trust-Evaluation Metric for Cloud Applications", International Journal of Machine Learning and Computing, Volume 1, Number 4, October 2011, pp. 416-421.

[13] Mohamed Firdhous, Osman Ghazali and Suhaidi Hassan, "Trust Management in Cloud Computing: A Critical Review”. International Journal on Advances in ICT for Emerging Regions, 2011, 04 (02): 24 - 36.

[14] R. Buyya et al., "Cloud computing and emerging IT platforms: Vision, hype, and reality for delivering computing as the 5th utility," Future Generation Computer Systems, vol. 25, no. 6, pp. 599-616, 2009.

[15] Supriya M, Venkataramana L.J, K Sangeeta and G K Patra, "Estimating Trust Value for Cloud Service Providers using Fuzzy Logic”. International Journal of Computer Applications, Volume 48- No.19, June 2012.

[16] Siani Pearson, "Privacy, Security and Trust in Cloud Computing”. HP Laboratories, Springer, June 2012.

[17] S. K. Garg, S. Versteeg, and R. Buyya, "A framework for ranking of cloud computing services," Future Generation Computer Systems, vol. 29, no. 4, pp. 1012 - 1023, 2013.

[18] Somesh Kumar Prajapati, Suvamoy Changder and Anirban Sarkar, "Trust Management Model For Cloud Computing Environment". Proceedings of the International Conference on Computing. Communication and Advanced Network ICCCAN 2013.

[19] Wickremasinghe, B, Calheiros R.N and Buyya, R. "CloudAnalyst: A CloudSim-Based Visual Modeller for Analyzing Cloud Computing Environments and Applications" 24th International Conference on Advanced Information Networking and Applications, Australia, April 2010, pp. 446-452.

[20] Xiaodong Sun, Guiran Chang and Fengyun Li. "A Trust Management Model to Enhance Security of Cloud Computing Environments", Second International Conference on Networking and Distributed Computing, China, September 2011, pp. 244-248. 
[21] Z. u. Rehman, O. K. Hussain, and F. K. Hussain, "Multi-criteria laaS service selection based on QoS history," in Proceedings of the 2013 IEEE 27th International Conference on Advanced Information Networking and Applications (AINA), pp. 1129-1135.

\section{Author' biography with Photo}

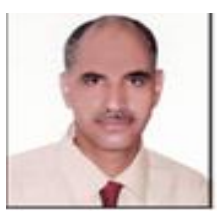

Hamdy M. Mousa: received the B.S. and M.S. in Electronic Engineering and Automatic control and measurements from Menoufia University, Faculty of Electronic Engineering in 1991 and 2002, respectively and received his $\mathrm{PhD}$ in Automatic control and measurements Engineering (Artificial intelligent) from Menoufia University, Faculty of Electronic in 2007. His research interest includes intelligent systems, Natural Language Processing, privacy, embedded systems, and GPS applications.

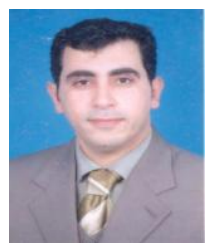

Gamal. F. Elhady: He completed his the B.S, M.S and Ph.D degree in Computer Science at Faculty of Science, in 1998 and 2006, Mansoura University, Egypt. During 1998 and 2006, he works a Demonstrator and Lecturer Assistance in Faculty of science computer science Dept. He is member of IAENG in USA (\# 108463). His research interest includes software programing, software testing, distributed system, data mining, database, Artificial intelligent, image processing and bioinformatics.

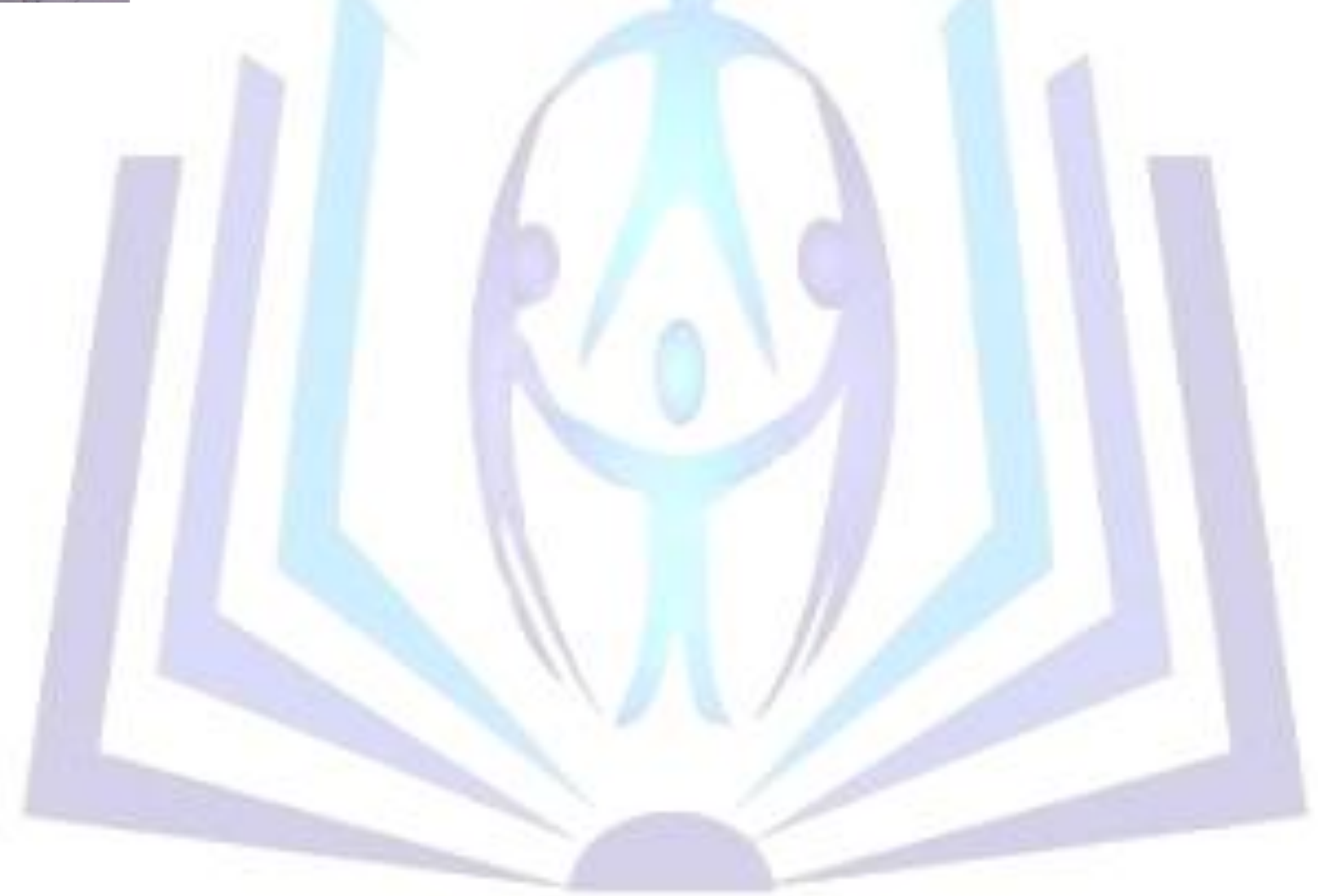

